\title{
Glucagon-Like Peptide-1, Diabetes, and Cognitive Decline: Possible Pathophysiological Links and Therapeutic Opportunities
}

\author{
Enrico Mossello, Elena Ballini, Marta Boncinelli, Matteo Monami, \\ Giuseppe Lonetto, Anna Maria Mello, Francesca Tarantini, Samuele Baldasseroni, \\ Edoardo Mannucci, and Niccolò Marchionni
}

Unit of Gerontology and Geriatric Medicine, Department of Critical Care Medicine and Surgery, University of Florence and Careggi Teaching Hospital, 50121 Florence, Italy

Correspondence should be addressed to Enrico Mossello, enrico.mossello@unifi.it

Received 25 February 2011; Accepted 5 April 2011

Academic Editor: Giovanni Di Pasquale

Copyright (C) 2011 Enrico Mossello et al. This is an open access article distributed under the Creative Commons Attribution License, which permits unrestricted use, distribution, and reproduction in any medium, provided the original work is properly cited.

\begin{abstract}
Metabolic and neurodegenerative disorders have a growing prevalence in Western countries. Available epidemiologic and neurobiological evidences support the existence of a pathophysiological link between these conditions. Glucagon-like peptide 1 (GLP-1), whose activity is reduced in insulin resistance, has been implicated in central nervous system function, including cognition, synaptic plasticity, and neurogenesis. We review the experimental researches suggesting that GLP-1 dysfunction might be a mediating factor between Type 2 diabetes mellitus (T2DM) and neurodegeneration. Drug treatments enhancing GLP-1 activity hold out hope for treatment and prevention of Alzheimer's disease (AD) and cognitive decline.
\end{abstract}

\section{Insulin Resistance and Cognitive Decline}

During the last years, Alzheimer's disease (AD) and clinical syndromes associated to insulin resistance have shown an ever-increasing prevalence in Western countries. These conditions pose a great threat to present and future population's health and represent two of the main causes of disability and health expenditures. Several research lines during the last decade have suggested an association among Type 2 diabetes mellitus (T2DM), insulin resistance, and cognitive decline, both in cross-sectional and in longitudinal studies.

Cross-sectional studies have found that older subjects with T2DM on average show a poorer cognitive performance than age-matched controls [1]. This association seems independent of other vascular risk factors and is attributable not only to a greater extent of white matter lesions but also to a more severe cortical atrophy [2], especially in temporomesial areas (hippocampus, amygdala) [3]. Moreover insulin resistance is associated to a worse cognitive performance in nondiabetic subjects too [4]. On the other hand crosssectional studies have observed a significant association of dementia, $\mathrm{AD}$ in particular, with T2DM [5] and insulin resistance [6].

Also several longitudinal studies have observed an association of T2DM with dementia risk over years [7]. Moreover it has been observed that older nondiabetic subjects with metabolic syndrome and increased level of inflammatory markers have an increased risk of subsequent cognitive decline [8]. Recently published data have shown that, among nondiabetic nondemented older subjects, insulin resistance is associated with $\mathrm{AD}$ incidence after a few years [9]. In keeping with this observation, insulin resistance has been associated recently with a greater extent of AD-like neuropathology at autopsy [10]. Therefore it is plausible that, among older subjects with asymptomatic $\mathrm{AD}$, a coexistent 
impairment of insulin metabolism can hasten symptoms expression.

This association might be linked to different biological mechanisms, first of all the presence of brain insulin resistance. In fact brain insulin receptor activity might have several neuroprotective effects, via PI3K (phosphatidylinositol-3-kinase)/Akt and ERK1/2 signalling pathways [11, 12]: decreased inflammation and apoptosis, increased synaptic plasticity, and inhibition of glycogen synthase kinase(GSK-)3, with subsequent decreased tau phosphorylation, which is a hallmark of AD neuropathology. It has been shown in vitro that insulin receptor activation is able to decrease synaptic binding sites through which amyloid oligomers produce their toxic activity, with resulting reduction of oxidative stress and dendritic spines loss [13]. Moreover postmortem analyses of $\mathrm{AD}$ patients brain have shown an impairment of insulin and IGF-1 receptors signalling, especially evident in neurons with neurofibrillary tangles, suggesting that degenerating neurons are resistant to insulin/IGF-1 action [14]. Some authors have even proposed the existence of a "Type 3 diabetes mellitus", limited to central nervous system (CNS), as a cause for $\mathrm{AD}$, as they were able to produce an AD-like neurodegeneration in a mouse model after intracerebroventricular injection of streptozotocin, inducing a depletion of CSF insulin without any change in peripheral insulin metabolism [15]. This hypothesis is supported by a pilot study, which has shown a significant cognitive improvement after intranasal insulin, without change of peripheral glucose metabolism [16].

On the other hand, experimental data have associated peripheral insulin resistance with reduced insulin activity inside the CNS, due to a reduced hormone transport through the blood-brain barrier [17], and with increased brain $\mathrm{A} \beta$ production in murine models of $\mathrm{AD}$ [18]. Moreover in studies of normal subjects with euglycemic clamp, the infusion of high insulin doses, mimicking insulin resistance, raises $A \beta-42$ levels, probably due to a reduced catabolism, and CNS inflammatory markers [19].

\section{Metabolic Effects of GLP-1, T2DM, and the "Gut-to-Brain" Axis}

Glucagon-like peptide-1 (GLP-1), a member of the incretins family, is a 30-aminoacid peptide, which is derived from preproglucagon molecule and is secreted by intestinal endocrine epithelial L-cells. It is the most potent stimulator of oral glucose-induced insulin secretion, it is released in response to meal intake and is rapidly metabolized and inactivated by dipeptidyl-peptidase-4 [20]. GLP-1 transmembrane receptor (GLP-1R) is a G-protein-coupled receptor and is expressed not only in pancreatic islets, but also in gastrointestinal tract, kidney, lung, vascular system, heart, and brain [21].

GLP-1R activation stimulates adenylate cyclase, with formation of cyclic adenosine monophosphate (cAMP) and subsequent phosphorylation of protein kinase A; moreover it activates PI3-kinase pathway, with downstream activation of Akt kinase, MAP-kinases, and src-kinases [22, 23]. Via these pathways, GLP-1 stimulates pancreatic $\beta$-cells, activating insulin secretion and inducing insulin gene expression [21]; moreover it has been shown that GLP-1 stimulates proliferation and differentiation, and reduces apoptosis of $\beta$ cells [23]. It seems interesting that, at least in pancreatic islets, GLP-1 activity seems synergic with insulin action in promoting $\beta$-cell survival [24].

Beyond its main activity, GLP-1 reduces plasma glucagon, inhibits gastrointestinal motility, and promotes satiety, reducing food intake [21]. Moreover it has a wide range of functions on glucose metabolism and cardiovascular system. In fact it improves insulin sensitivity, reduces appetite, modulates heart rate and blood pressure, reduces vascular tone, ameliorates endothelial function, and increases myocardial contractility, with preliminary data suggesting clinical benefit in heart failure [25].

It has been known for many years that T2DM is characterized by a severely reduced incretin effect, defined as the difference between insulin responses to oral and intravenous glucose administration [26]. Reduced GLP-1 levels have been observed after a mixed meal in Type 2 diabetes compared with controls [27], with a marked reduction especially of the late-phase response [28]. Moreover an altered GLP-1 response both to mixed meal [29] and to oral glucose load [30] has been observed in insulin resistance.

It has been proven that at least part of the metabolic effect of GLP-1 is mediated by CNS [31]. In fact brain GLP$1 \mathrm{R}$ are partly responsible not only for food intake control, but also for control of glucose homeostasis, with coordinate actions on pancreas and liver [32]. It has been observed in mice that GLP-1 secreted into the hepatoportal vein increases the firing rate of the vagus nerve, sending signals to the brainstem nucleus of the tractus solitarius, which on the other hand releases GLP-1 in hypothalamic regions, inducing reflex insulin secretion and muscle glycogen synthesis [33]. These data support the existence of a "gut-to-brain axis", with a central role of GLP-1 released both by intestinal cells and by neurons, involved in the regulation of systemic glucose metabolism, whose activity seems to be blunted in high-fat fed, insulin-resistant mice [33].

On the other hand, it has been hypothesized that GLP1 can influence brain metabolism. In fact a small human study with FDG-PET (positron emission tomography with 18-fluorodeoxyglucose) has shown a possible effect of GLP-1 on brain glucose metabolism. In this study GLP-1 infusion in normoglycemic conditions reduced glucose transport across blood-brain barrier in specific brain areas while a trend of decrease of cerebral metabolic rate was also observed, thus maintaining brain glucose concentration unchanged. This observation leads the authors to hypothesize that GLP-1 may exert a neuroprotective effect by limiting intracerebral glucose fluctuation in postprandial periods, when plasma glucose is increased [34].

\section{GLP-1, Neuroprotection, and Alzheimer's Disease}

Beyond its metabolic role, several studies have clarified a role of GLP-1 in CNS function. Experimental studies have identified a widespread expression of GLP-1R across a large number of rat brain regions, not directly involved 
in metabolic control, including hippocampus, thalamus, striatum, substantia nigra, amygdala, nucleus basalis Meynert, subventricular zone, and temporal cortex [35]. GLP-1R expression has been observed in specific cellular subtypes which are crucial for memory and learning functions, including pyramidal neurons of CA region and granule cells of dentate gyrus in hippocampus, and in large neocortical neurons [36]. Other authors have observed GLP-1R expression also on glial cells (microglia and astrocytes), proposing a role for them as modulators of CNS inflammation [37].

The neurotrophic effect of GLP-1R has been strongly suggested by studies of mice knockout (KO) for GLP-1R, which show an impairment of contextual memory, as assessed by the passive avoidance test, which measures the ability of the animal to learn and remember that an instinctive behavior causes a punishment. Memory impairment of KO mice was reversible after GLP1-R gene DNA transfer with a viral vector [38]. These data were confirmed in a subsequent study of cognitive functions in a GLP-1R KO mouse model: a reduced recognition memory and spatial memory has been shown while other behavioural parameters, including exploration and anxiety, were unchanged. Interestingly a neurophysiological study of hippocampus CA1 area mice showed a severe impairment of long-term potentiation, which is the synaptic process associated to consolidation of long-term memory [39].

Adding to these observations, it has been demonstrated that GLP-1 analogues, which have greater metabolic stability than the native molecule, also cross blood-brain barrier when administered peripherally [40]. As only small amounts of native GLP-1 reach CNS if peripherally administered, due to rapid catabolism, much of the pharmacologic research has focused on analogues of the molecule, which are more resistant to degradation, while retaining the stimulatory effect on GLP-1R.

Several experimental evidences have demonstrated a neuroprotective role for GLP-1 and its analogues. In cultured rat pheochromocytoma cells, some authors observed that GLP-1 and exendin-4, (Ex-4) a long-acting GLP-1 analogue, stimulated neurite outgrowth in a similar fashion to nerve-growth-factor (NGF). Besides, $E x-4$ was able to augment NGF-induced neuronal differentiation, and apparently attenuated neural degeneration following NGF withdrawal [41]. Other authors confirmed these data on cultured neural cells, finding that GLP-1 exposure protected cells from death promoted by NGF deprivation, by suppressing the proapoptotic protein $\mathrm{Bim}(\mathrm{Bcl}-2$ interacting mediator of cell death) [42].

Part of the neuroprotective effect of GLP-1R agonists is probably related to reduced neuronal damage due to amyloid metabolism. In fact $E x-4$ has been shown to reduce the synthesis of amyloidogenic $\mathrm{A} \beta$ fragment and to protect cells from $\beta$-amyloid toxicity in cultured neural cells [43]. Moreover intracerebroventricular injection of GLP-1 or Ex-4 has been shown to decrease levels of brain amyloid fragment in control mice [44].

The efficacy of peripherally administered GLP-1 analogues has been shown also in experimental models. In normal adult rats Ex-4 improves hippocampus-based cognitive performance, namely, spatial learning and working memory, as assessed by the "radial arm maze," which allows the measurement of the time necessary for the animal to find food, placed at the end of several equidistantly spaced arms, which radiate form a central platform [45]. In the same paper the repeated administration of Ex-4 was effective in ameliorating mood and reducing hopelessness, as measured by the immobility time in the "forced swim test," during which animals are forced to swim in a cylinder filled with water, from which they cannot escape [45].

The previously mentioned behavioural effects are paralleled by several histochemical changes observed "ex vivo." Intraperitoneal administration of Ex-4 has increased both the number of proliferating cells and the expression of neuronal differentiation markers in adult rat hippocampus and in subventricular zone $[45,46]$.

A neuroprotective effect of $E x-4$ has been observed recently in experimental models of neurodegeneration. In the triple transgenic $\mathrm{AD}$-mouse, which is an experimental model of the human disease, the induction of diabetes with streptozotocin was associated with an increase of $\beta$-amyloid brain load, consistently with evidence linking T2DM with $\mathrm{AD}$ neuropathology, and subcutaneous administration of Ex-4 prevented this increase [43]. The results of this study suggests that Ex-4 may have a therapeutic role in $\mathrm{AD}$, alone or with T2DM. Moreover in an animal model of Parkinson's disease, in which Ex-4 was able to increase the number of dopaminergic neurons in the substantia nigra, a contemporary reduction of extrapyramidal signs was observed [46].

Another long-acting GLP-1 analogue used for T2DM, liraglutide ( $\mathrm{Lir}$ ), is able to cross the blood-brain barrier [47], and has shown neuroprotective effects in experimental models. This is also the case for other GLP-1 mimetics, Asp(7)GLP-1, N-glyc-GLP-1, and Pro(9)GLP-1, which, like Lir, are able to increase synaptic plasticity, measured as longterm potentation in CA1 hippocampal region of rats [47].

Both Ex-4 and Lir were recently tested for their neuroprotective effect in mouse models of T2DM. In this study GLP-1 analogues were injected subcutaneously to three mouse models of diabetes (ob/ob mice, $\mathrm{db} / \mathrm{db}$ mice, and high-fat-diet-fed mice). At the histochemical analysis a greater number of proliferating neurons in hippocampal dentate gyrus was found in diabetic mice compared with nondiabetic controls, and this number was further enhanced by both drugs [48]. The increased neurogenesis in T2DM models was interpreted by the authors as a response to increased brain cell death which is associated with the disease; this compensatory process would be supported by GLP-1 mimetics. This interpretation is supported by another paper published by the same authors, regarding the cognitive effect of Lir in the mouse model of high-fat-dietinduced obesity. In parallel with metabolic changes (weight loss, increased glucose tolerance), mice treated with $\mathrm{Lir}$ subcutaneous injections showed an improvement of learning and memory ability, assessed with "object recognition test." The test measures the extent of exploratory activity of a previously presented object, which is expected to be lower in comparison with newly presented objects, and is therefore 
considered a measure of recognition memory. Furthermore, Lir reduced negative effects of high-fat diet on hippocampal long-term potentation [49].

Another analogue of GLP-1, Val(8)-GLP-1(7-36), was studied in rats, and its intracerebroventricular injection reversed the impairment of spatial memory induced by injection of $\beta$-amyloid fragment $A \beta 1-40$. Moreover pretreatment with Val8-glucagon-like peptide-1 prevented the impairment of hippocampal long-term potentiation that is induced by the presence of $\mathrm{A} \beta 1-40$ [50]. These data are consistent with a different research, performed with the same molecule, on AD-like mice with a double mutation of amyloid precursor protein (APP) and presenilin 1 (PS1). A beneficial effect was observed on long-term potentiation, both in young (9 months) and in older animals (18 months) while $\beta$ amyloid plaques and inflammatory microglia activation was unchanged in treated animals [51]. These data support the hypothesis that GLP-1R agonists might partly prevent toxic effect of $\beta$-amyloid deposition, with obvious interest for possible $\mathrm{AD}$ treatment.

With the background of the previously discussed preclinical data, $E x-4$ is now being studied as a treatment for $\mathrm{AD}$ and PD in Phase 2 studies (see http://www.clinicaltrials.gov/, NCT01255163 and NCT01174810). Of notice, the hypoglycemic effect of GLP-1 analogues in normoglycemic subjects seems minimal $[52,53]$ and should not constitute a major concern for the treatment of nondiabetic subjects.

\section{Conclusions}

The available evidences strongly support the hypothesis that the observed association between insulin resistance/T2DM and cognitive decline/dementia is mediated not only by wellknown vascular changes, but also by direct neurotoxic effect of glucose metabolism impairment.

Incretin activity, and GLP-1 in particular, which is reduced in insulin resistance conditions, represents a possible pathophysiological link between metabolism disorders and neurodegeneration. The reduction of GLP-1 levels, characteristic of T2DM, might be associated to a reduced neuroprotection, which seems particularly relevant for hippocampal regions [39], where $\mathrm{AD}$ neuropathology is most evident. It is tempting to speculate that GLP-1 and insulin have synergistic activity in promoting neuron survival, as it has been shown in pancreatic $\beta$-cells, both for native GLP-1 [24] and for GLP1 synthetic analogues $[54,55]$. Human studies evaluating the association between GLP-1 levels and cognitive function, controlling for insulin resistance status, are needed to support the hypothesis of a direct neuroprotective effect of incretins.

GLP-1 analogues has been shown to enhance cognitive function in control animals $[38,45]$, to prevent cognitive impairment in models of T2DM $[48,49]$, and to counteract $\beta$-amyloid toxicity in models of $\mathrm{AD}[43,51]$. These experimental data support the effort of testing such molecules both for the prevention of cognitive decline in T2DM and for treatment of $\mathrm{AD}$ patients in randomized controlled trials.

\section{References}

[1] G. Logroscino, J. H. Kang, and F. Grodstein, "Prospective study of type 2 diabetes and cognitive decline in women aged 70-81 years," British Medical Journal, vol. 328, no. 7439, pp. 548-551, 2004.

[2] S. M. Manschot, G. J. Biessels, H. De Valk et al., "Metabolic and vascular determinants of impaired cognitive performance and abnormalities on brain magnetic resonance imaging in patients with type 2 diabetes," Diabetologia, vol. 50, no. 11, pp. 2388-2397, 2007.

[3] T. Den Heijer, S. E. Vermeer, E. J. Van Dijk et al., "Type 2 diabetes and atrophy of medial temporal lobe structures on brain MRI," Diabetologia, vol. 46, no. 12, pp. 1604-1610, 2003.

[4] S. Kalmijn, E. J. M. Feskens, L. J. Launer, T. Stijnen, and D. Kromhout, "Glucose intolerance, hyperinsulinaemia and cognitive function in a general population of elderly men," Diabetologia, vol. 38, no. 9, pp. 1096-1102, 1995.

[5] A. Ott, R. P. Stolk, A. Hofman, F. Van Harskamp, D. E. Grobbee, and M. M. B. Breteler, "Association of diabetes mellitus and dementia: the Rotterdam Study," Diabetologia, vol. 39, no. 11, pp. 1392-1397, 1996.

[6] J. Kuusisto, K. Koivisto, L. Mykkänen et al., "Association between features of the insulin resistance syndrome and Alzheimer's disease independently of apolipoprotein E4 phenotype: cross sectional population based study," British Medical Journal, vol. 315, no. 7115, pp. 1045-1049, 1997.

[7] G. J. Biessels, S. Staekenborg, E. Brunner, C. Brayne, and P. Scheltens, "Risk of dementia in diabetes mellitus: a systematic review," Lancet Neurology, vol. 5, no. 1, pp. 64-74, 2006.

[8] K. Yaffe, A. Kanaya, K. Lindquist et al., "The metabolic syndrome, inflammation, and risk of cognitive decline," Journal of the American Medical Association, vol. 292, no. 18, pp. 2237-2242, 2004.

[9] E. M. C. Schrijvers, J. C. M. Witteman, E. J. G. Sijbrands, A. Hofman, P. J. Koudstaal, and M. M. B. Breteler, "Insulin metabolism and the risk of Alzheimer disease: the Rotterdam Study," Neurology, vol. 75, no. 22, pp. 1982-1987, 2010.

[10] T. Matsuzaki, K. Sasaki, Y. Tanizaki et al., "Insulin resistance is associated with the pathology of Alzheimer disease: the Hisayama study," Neurology, vol. 75, no. 9, pp. 764-770, 2010.

[11] S. Cardoso, S. Correia, R. X. Santos et al., "Insulin is a twoedged knife on the brain," Journal of Alzheimer's Disease, vol. 18, no. 3, pp. 483-507, 2009.

[12] L. Plum, M. Schubert, and J. C. Brüning, "The role of insulin receptor signaling in the brain," Trends in Endocrinology and Metabolism, vol. 16, no. 2, pp. 59-65, 2005.

[13] F. G. De Felice, M. N. N. Vieira, T. R. Bomfim et al., "Protection of synapses against Alzheimer's-linked toxins: insulin signaling prevents the pathogenic binding of $\mathrm{A} \beta$ oligomers," Proceedings of the National Academy of Sciences of the United States of America, vol. 106, no. 6, pp. 1971-1976, 2009.

[14] A. M. Moloney, R. J. Griffin, S. Timmons, R. O'Connor, R. Ravid, and C. O'Neill, "Defects in IGF-1 receptor, insulin receptor and IRS-1/2 in Alzheimer's disease indicate possible resistance to IGF-1 and insulin signalling," Neurobiology of Aging, vol. 31, no. 2, pp. 224-243, 2010.

[15] N. Lester-Coll, E. J. Rivera, S. J. Soscia, K. Doiron, J. R. Wands, and S. M. De La Monte, "Intracerebral streptozotocin model of type 3 diabetes: relevance to sporadic Alzheimer's disease," Journal of Alzheimer's Disease, vol. 9, no. 1, pp. 13-33, 2006. 
[16] M. A. Reger, G. S. Watson, P. S. Green et al., "Intranasal insulin improves cognition and modulates $\beta$-amyloid in early AD," Neurology, vol. 70, no. 6, pp. 440-448, 2008.

[17] S. Craft, "Insulin resistance syndrome and Alzheimer's disease: age- and obesity-related effects on memory, amyloid, and inflammation," Neurobiology of Aging, vol. 26, supplement 1, pp. S65-S69, 2005.

[18] L. Ho, W. Qin, P. N. Pompl et al., "Diet-induced insulin resistance promotes amyloidosis in a transgenic mouse model of Alzheimer's disease," The FASEB Journal, vol. 18, no. 7, pp. 902-904, 2004.

[19] M. A. Fishel, G. S. Watson, T. J. Montine et al., "Hyperinsulinemia provokes synchronous increases in central inflammation and $\beta$-amyloid in normal adults," Archives of Neurology, vol. 62, no. 10, pp. 1539-1544, 2005.

[20] Z. Wang, R. M. Wang, A. A. Owji, D. M. Smith, M. A. Ghatei, and S. R. Bloom, "Glucagon-like peptide-1 is a physiological incretin in rat," Journal of Clinical Investigation, vol. 95, no. 1, pp. 417-421, 1995.

[21] J. J. Holst, "The physiology of glucagon-like peptide 1," Physiological Reviews, vol. 87, no. 4, pp. 1409-1439, 2007.

[22] H. Hui, A. Nourparvar, X. Zhao, and R. Perfetti, "Glucagonlike peptide-1 inhibits apoptosis of insulin-secreting cells via a cyclic $5^{\prime}$-adenosine monophosphate-dependent protein kinase A- and a phosphatidylinositol 3-kinase-dependent pathway," Endocrinology, vol. 144, no. 4, pp. 1444-1455, 2003.

[23] P. L. Brubaker and D. J. Drucker, "Minireview: glucagonlike peptides regulate cell proliferation and apoptosis in the pancreas, gut, and central nervous system," Endocrinology, vol. 145, no. 6, pp. 2653-2659, 2004.

[24] U. S. Jhala, G. Canettieri, R. A. Screaton et al., "cAMP promotes pancreatic $\beta$-cell survival via CREB-mediated induction of IRS2," Genes and Development, vol. 17, no. 13, pp. 15751580, 2003.

[25] D. J. Grieve, R. S. Cassidy, and B. D. Green, "Emerging cardiovascular actions of the incretin hormone glucagon-like peptide-1: potential therapeutic benefits beyond glycaemic control?" British Journal of Pharmacology, vol. 157, no. 8, pp. 1340-1351, 2009.

[26] M. Nauck, F. Stockmann, R. Ebert, and W. Creutzfeldt, "Reduced incretin effect in Type 2 (non-insulin-dependent) diabetes," Diabetologia, vol. 29, no. 1, pp. 46-52, 1986.

[27] E. Mannucci, A. Ognibene, F. Cremasco et al., "Glucagon-like peptide (GLP)-1 and leptin concentrations in obese patients with Type 2 diabetes mellitus," Diabetic Medicine, vol. 17, no. 10, pp. 713-719, 2000.

[28] T. Vilsbøll, T. Krarup, C. F. Deacon, S. Madsbad, and J. J. Holst, "Reduced postprandial concentrations of intact biologically active glucagon-like peptide 1 in type 2 diabetic patients," Diabetes, vol. 50, no. 3, pp. 609-613, 2001.

[29] E. Rask, T. Olsson, S. Söderberg et al., "Impaired incretin response after a mixed meal is associated with insulin resistance in nondiabetic men," Diabetes Care, vol. 24, no. 9, pp. 1640-1645, 2001.

[30] E. Rask, T. Olsson, S. Söderberg et al., "Insulin secretion and incretin hormones after oral glucose in non-obese subjects with impaired glucose tolerance," Metabolism, vol. 53, no. 5, pp. 624-631, 2004.

[31] R. Burcelin, M. Serino, and C. Cabou, "A role for the gut-tobrain GLP-1-dependent axis in the control of metabolism," Current Opinion in Pharmacology, vol. 9, no. 6, pp. 744-752, 2009.

[32] D. A. Sandoval, D. Bagnol, S. C. Woods, D. A. D’Alessio, and R. J.Seeley, "Arcuate glucagon-like peptide 1 receptors regulate glucose homeostasis but not food intake," Diabetes, vol. 57, no. 8, pp. 2046-2054, 2008.

[33] C. Knauf, P. D. Cani, D. H. Kim et al., "Role of central nervous system glucagon-like peptide-1 receptors in enteric glucose sensing," Diabetes, vol. 57, no. 10, pp. 2603-2612, 2008.

[34] S. Lerche, B. Brock, J. Rungby et al., "Glucagon-like peptide1 inhibits blood-brain glucose transfer in humans," Diabetes, vol. 57, no. 2, pp. 325-331, 2008.

[35] I. Merchenthaler, M. Lane, and P. Shughrue, "Distribution of pre-pro-glucagon and glucagon-like peptide-1 receptor messenger RNAs in the rat central nervous system," Journal of Comparative Neurology, vol. 403, no. 2, pp. 261-280, 1999.

[36] A. Hamilton and C. Hölscher, "Receptors for the incretin glucagon-like peptide-1 are expressed on neurons in the central nervous system," NeuroReport, vol. 20, no. 13, pp. 1161-1166, 2009.

[37] T. Iwai, S. Ito, K. Tanimitsu, S. Udagawa, and J. I. Oka, "Glucagon-like peptide-1 inhibits LPS-induced IL- $1 \beta$ production in cultured rat astrocytes," Neuroscience Research, vol. 55, no. 4, pp. 352-360, 2006.

[38] M. J. During, L. Cao, D. S. Zuzga et al., "Glucagon-like peptide-1 receptor is involved in learning and neuroprotection," Nature Medicine, vol. 9, no. 9, pp. 1173-1179, 2003.

[39] T. Abbas, E. Faivre, and C. Hölscher, "Impairment of synaptic plasticity and memory formation in GLP-1 receptor KO mice: interaction between type 2 diabetes and Alzheimer's disease," Behavioural Brain Research, vol. 205, no. 1, pp. 265-271, 2009.

[40] A. J. Kastin and V. Akerstrom, "Entry of exendin-4 into brain is rapid but may be limited at high doses," International Journal of Obesity, vol. 27, no. 3, pp. 313-318, 2003.

[41] T. Perry, D. K. Lahiri, D. Chen et al., "A novel neurotrophic property of glucagon-like peptide 1: a promoter of nerve growth factor-mediated differentiation in PC12 cells," Journal of Pharmacology and Experimental Therapeutics, vol. 300, no. 3, pp. 958-966, 2002.

[42] S. C. Biswas, J. Buteau, and L. A. Greene, "Glucagonlike peptide-1 (GLP-1) diminishes neuronal degeneration and death caused by NGF deprivation by suppressing Bim induction," Neurochemical Research, vol. 33, no. 9, pp. 18451851, 2008.

[43] Y. Li, K. B. Duffy, M. A. Ottinger et al., "GLP-1 receptor stimulation reduces amyloid- $\beta$ peptide accumulation and cytotoxicity in cellular and animal models of Alzheimer's disease," Journal of Alzheimer's Disease, vol. 19, no. 4, pp. 12051219, 2010.

[44] T. Perry, D. K. Lahiri, K. Sambamurti et al., "Glucagonlike peptide- 1 decreases endogenous amyloid- $\beta$ peptide $(\mathrm{A} \beta)$ levels and protects hippocampal neurons from death induced by A $\beta$ and iron," Journal of Neuroscience Research, vol. 72, no. 5, pp. 603-612, 2003.

[45] R. Isacson, E. Nielsen, K. Dannaeus et al., "The glucagonlike peptide 1 receptor agonist exendin- 4 improves reference memory performance and decreases immobility in the forced swim test," European Journal of Pharmacology, vol. 650, no. 1, pp. 249-255, 2011.

[46] G. Bertilsson, C. Patrone, O. Zachrisson et al., "Peptide hormone exendin-4 stimulates subventricular zone neurogenesis in the adult rodent brain and induces recovery in an animal model of Parkinson's disease," Journal of Neuroscience Research, vol. 86, no. 2, pp. 326-338, 2008.

[47] P. L. McClean, V. A. Gault, P. Harriott, and C. Hölscher, "Glucagon-like peptide-1 analogues enhance synaptic plasticity in the brain: a link between diabetes and Alzheimer's 
disease," European Journal of Pharmacology, vol. 630, no. 1-3, pp. 158-162, 2010.

[48] A. Hamilton, S. Patterson, D. Porter, V. A. Gault, and C. Holscher, "Novel GLP-1 mimetics developed to treat type 2 diabetes promote progenitor cell proliferation in the brain," Journal of Neuroscience Research, vol. 89, no. 4, pp. 481-489, 2011.

[49] D. W. Porter, B. D. Kerr, P. R. Flatt, C. Holscher, and V. A. Gault, "Four weeks administration of Liraglutide improves memory and learning as well as glycaemic control in mice with high fat dietary-induced obesity and insulin resistance," Diabetes, Obesity and Metabolism, vol. 12, no. 10, pp. 891-899, 2010.

[50] X. H. Wang, L. Li, C. Hölscher, Y. F. Pan, X. R. Chen, and J. S. Qi, "Val8-glucagon-like peptide-1 protects against A $\beta 1$ 40-induced impairment of hippocampal late-phase long-term potentiation and spatial learning in rats," Neuroscience, vol. 170, no. 4, pp. 1239-1248, 2010.

[51] S. Gengler, P. L. McClean, R. McCurtin, V. A. Gault, and C. Hölscher, "Val(8)GLP-1 rescues synaptic plasticity and reduces dense core plaques in APP/PS1 mice," Neurobiology of Aging. In press.

[52] D. J. Grieve, R. S. Cassidy, and B. D. Green, "Emerging cardiovascular actions of the incretin hormone glucagon-like peptide-1: potential therapeutic benefits beyond glycaemic control?" British Journal of Pharmacology, vol. 157, no. 8, pp. 1340-1351, 2009.

[53] M. A. Nauck, M. M. Heimesaat, K. Behle et al., "Effects of glucagon-like peptide 1 on counterregulatory hormone responses, cognitive functions, and insulin secretion during hyperinsulinemic, stepped hypoglycemic clamp experiments in healthy volunteers," Journal of Clinical Endocrinology and Metabolism, vol. 87, no. 3, pp. 1239-1246, 2002.

[54] S. Park, X. Dong, T. L. Fisher et al., "Exendin-4 uses Irs2 signaling to mediate pancreatic $\beta$ cell growth and function," Journal of Biological Chemistry, vol. 281, no. 2, pp. 1159-1168, 2006.

[55] S. Park, S. M. Hong, and SO. R. Sung, "Exendin-4 and exercise promotes $\beta$-cell function and mass through IRS2 induction in islets of diabetic rats," Life Sciences, vol. 82, no. 9-10, pp. 503 511,2008 . 


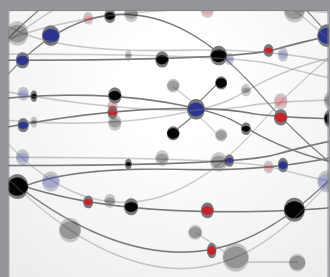

The Scientific World Journal
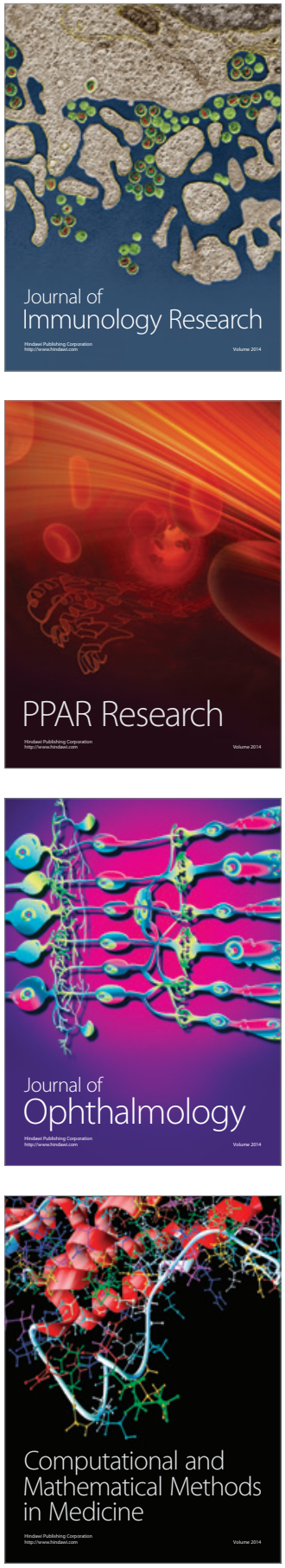

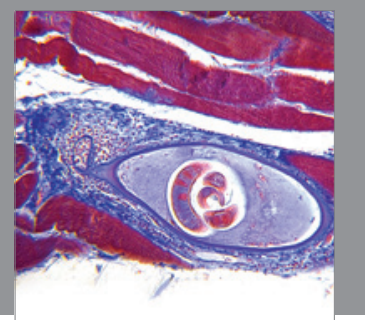

Gastroenterology

Research and Practice
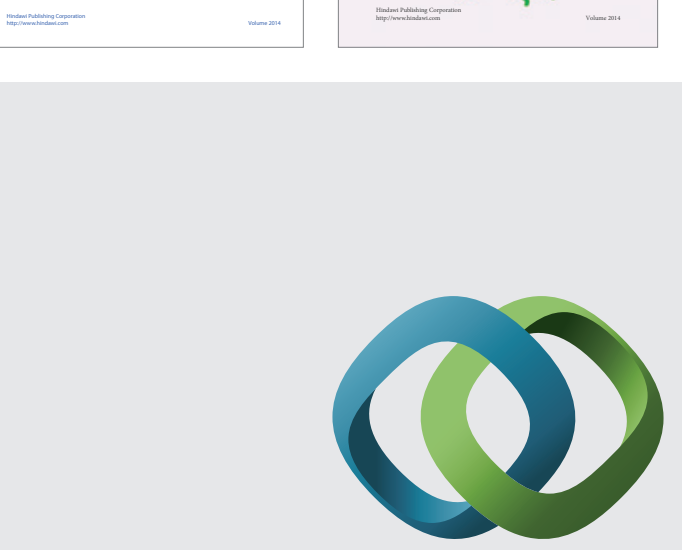

\section{Hindawi}

Submit your manuscripts at

http://www.hindawi.com
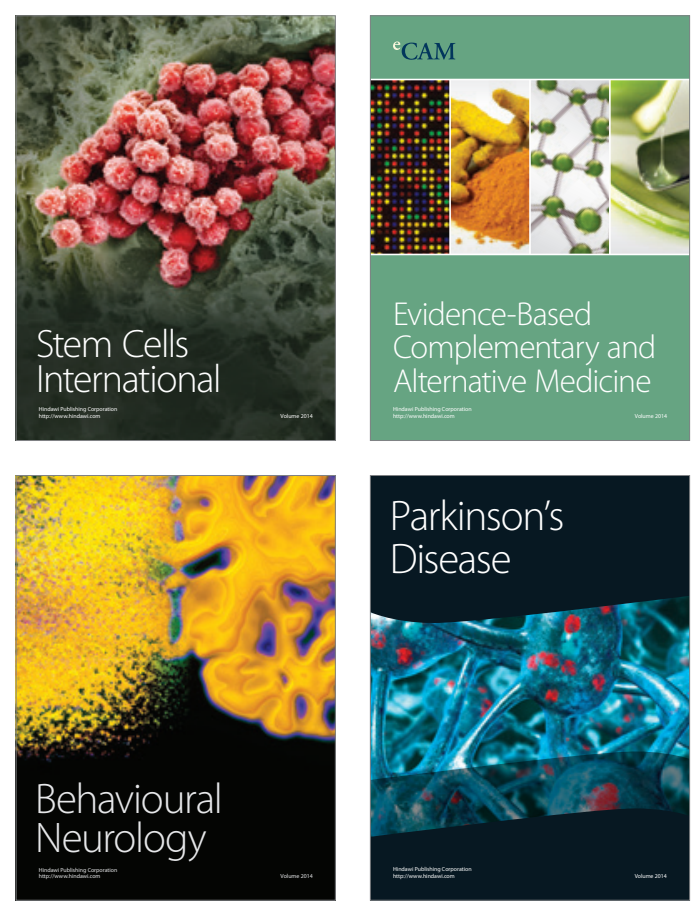

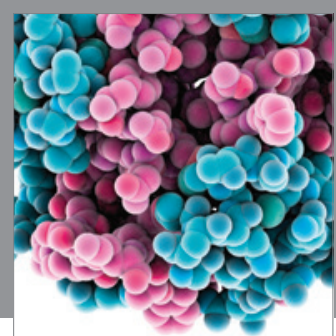

Journal of
Diabetes Research

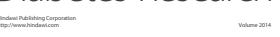

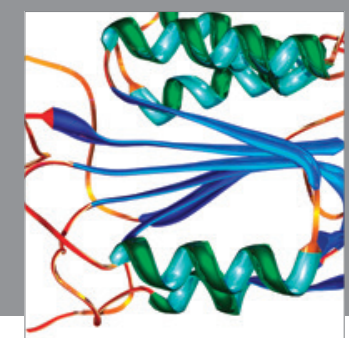

Disease Markers
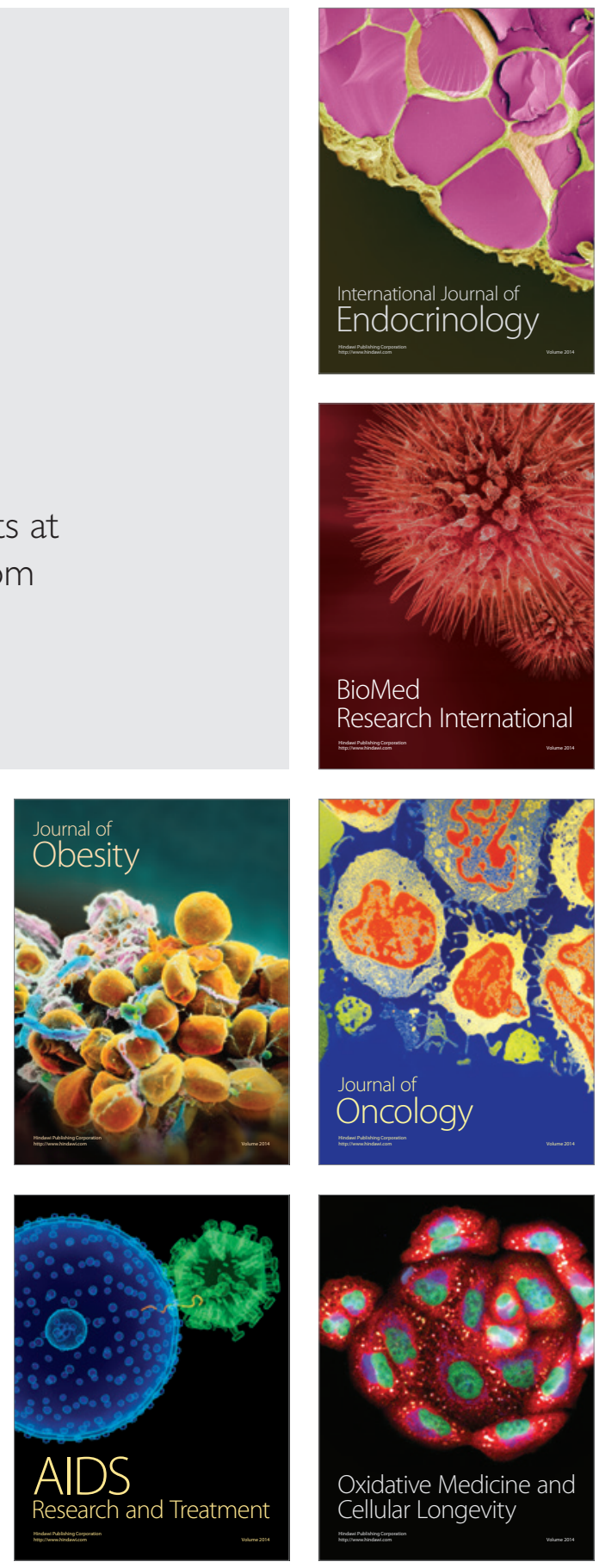